\title{
Ethical considerations in the design and conduct of a cluster-randomised mycotoxin mitigation trial in Tanzania
}

\author{
E. Phillips ${ }^{1 \text { ", P.C. Turner }}{ }^{2}$, F.M. Ngure ${ }^{3}$, N. Kassim ${ }^{4}$, E. Makule ${ }^{4}$, L.E. Smith ${ }^{5}$, R.J. Nelson ${ }^{6}$ and R.J. Stoltzfus ${ }^{7}$ \\ ${ }^{1}$ Division of Nutritional Sciences, Cornell University, 244 Garden, Ithaca, NY 14853, USA; ${ }^{2}$ MIAEH, School of Public \\ Health, University of Maryland, College Park, MD 20740, USA; ${ }^{3}$ Independent Research Consultant, Arusha, Tanzania \\ and Nairobi, Kenya; ${ }^{4}$ Department of Food Biotechnology and Nutritional Sciences, (FBNS), School of Life Science and \\ Bio-Engineering, The Nelson Mandela African Institution of Science and Technology (NM-AIST), P.O. Box 447, Arusha, \\ Tanzania; ${ }^{5}$ Department of Population Medicine and Diagnostics, Cornell University, Ithaca, NY 14853, USA; ${ }^{6}$ School of \\ Integrative Plant Science and Department of Global Development, Cornell University, Ithaca, NY 14853, USA; ${ }^{7}$ Goshen \\ College, 1700 S. Main Street, Goshen, Indiana 46526, USA; elp28@cornell.edu
}

Received: 21 April 2021 / Accepted: 29 November 2021

(c) 2022 Wageningen Academic Publishers

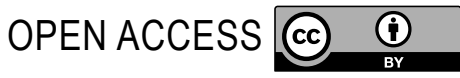

OPINION ARTICLE

\begin{abstract}
Aflatoxins are fungal metabolites that commonly contaminate staple food crops in tropical regions. Acute aflatoxin consumption in very high concentration causes aflatoxicosis and acute liver failure, while chronic, moderate levels of intake cause hepatocellular carcinoma. The effects of frequent moderate- to high-level exposure during infancy, however, is less clearly understood. Half a billion people in low- and middle-income countries continue to be exposed to aflatoxins through dietary consumption, in part because of lack of enforcement of regulatory limits and few feasible long-term mitigation options in these settings. Several epidemiologic studies have shown an association between aflatoxin exposure in infants and young children and growth failure, but strong experimental evidence is lacking. The Mycotoxin Mitigation Trial conducted in Tanzania was a cluster-randomised trial to assess the effect of a reduced aflatoxin diet on linear growth. Prior to the design and implementation of this trial, a group of multidisciplinary and multi-national scientists reviewed literature in biomedical, public health, environmental health ethics. In this paper we outline the most salient ethical questions and dilemmas in the potential conduct of such a study and describe the ethical precedents and principles that informed our decision-making processes and ultimate study protocol.
\end{abstract}

Keywords: aflatoxin, bioethics, infant growth

\section{Introduction}

Research conducted with human subjects is informed and regulated by multiple guidelines, codes of practice and declarations including The Nuremburg Code, Declaration of Helsinki, Belmont Report, International Ethical Guidelines for Biomedical Research involving Human Subjects and Good Clinical Practice guidelines (Council for International Organizations of Medical Sciences (CIOMS), 2016; International Council for Harmonization of Technical Requirements for Registration of Pharmaceuticals for Human Use (ICH), 1997; Katz, 1996; The National Commission for the Protection of Human Subjects of
Biomedical and Behavioral Research, 1979; World Medical Association, 2013). Many of the basic principles described in these documents are codified into national laws. Relying on these guidelines and laws, national and institutional ethical review boards are empowered to determine the ethical acceptability and permissibility of specific research design, conduct and analysis.

Even with extensive guidance on the conduct of human subject research, debates and quandaries in research ethics arise. These debates often highlight the vastly divergent contexts in which research is carried out around the world. Researchers are challenged to know whether and 
how to account for these contextual differences, especially when those differences generate the need for research. Different fields of study have divergent, possibly conflicting objectives. For example, clinicians have a duty to protect individual patients whereas public health researchers are concerned for the health of entire populations and communities. Debates also arise as researchers are pushing boundaries of conventional research methods to understand and find solutions to complex, multi-faceted problems including climate change, poverty, food security, and food safety in a globally integrated food system.

Research into mycotoxins and human health encompasses all of these challenges. Understanding and controlling hazardous substances in our food system is a critical goal for agricultural scientists, nutritionists, toxicologists and microbiologists. Dietary staples, such as grains and nuts, are frequently contaminated with secondary metabolites of fungal colonisation. When toxic these metabolites are called mycotoxins. Aflatoxins (AF) are an important family of mycotoxins that most commonly contaminate maize and groundnut, during both the growth of crops in the field and again during storage. In high-income countries, AF is well-regulated and managed throughout the value chain, although at high cost. In the United States, for example, the cost of control, surveillance and loss of food crops due to AF are estimated to be $\$ 47$ million for maize and groundnuts annually (Vardon et al., 2003).

In the face of such costs, and lacking strong regulatory infrastructures and feasible interventions to mitigate toxins, around 500 million people remain at risk of being chronically exposed to dietary AF at high levels in lowand middle-income countries (LMICs) (Pitt et al., 2012; Visser et al., 2020; Wu et al., 2014a). Low-resource and rural communities typically have restricted dietary diversity, and tend to be at greatest risk of contaminated food supplies (Pitt et al., 2012; Wild et al., 2015). Chronic AF consumption can lead to liver cancer and ingestion of very high levels causes acute toxicosis and possibly death (IARC, 2002; Lewis et al., 2005; Williams et al., 2004).

There is now a significant body of literature assessing the effect of AF consumption on infant growth. A 2019 systematic review identified two randomised controlled trials (RCT) and 29 observational and prospective cohort studies studying this relationship, but the authors concluded that the overall quality of evidence was low due to risk of bias and inconsistency in the reported outcomes, measurement method and exposure period, as well as failure to adjust for confounders (Tesfamariam et al., 2019). Our internal review found that regions with the highest levels of AF contamination and intake, multiple studies have shown a positive association between AF exposure and growth faltering, suggesting a significant dose-response relationships and a possible threshold of effect (Gong et al., 2002, 2003, 2004; Mahfuz et al., 2020; Mitchell et al., 2017; Turner et al., 2007; Watson et al., 2018). To generate strong causal evidence for the relationship between AF and stunting, we conducted a cluster-randomised trial (CRT) to assess the effect of AF on linear growth in infants and young children (Phillips et al., 2020).

\section{Unique ethical dilemmas in this study}

As with any research on human subjects, we received ethical approval for the study through Cornell's Institutional Review Board and Tanzania's National Institute for Medical Research. Both bodies approved the research application following minor clarifications in study protocol. In accordance with Good Clinical Practices, we formed a Data Safety and Monitoring Board (DSMB), comprised of a Tanzanian biostatistician, nutritionist and paediatrician. We shared anthropometric data with the DSMB every six months during the trial, as well as immediate notice of any adverse events.

However, because this research involved exposure to toxins in a low-resource setting, we felt it was necessary to go beyond the typical ethical approvals at multiple points throughout the planning process. We initiated consultations with other researchers working in related fields and performed a literature review focused in biomedical, public health and environmental health ethics to identify the most salient ethical complexities and questions for this study. Below we describe four key questions pertinent to this research study and share our responses to them.

Question 1: Given what is already known about the health effects of $A F$, should a question about the relationship between $A F$ and child growth be studied further in human subjects?

Human subject research must meet certain ethical criteria to be conducted. These include: (1) social and/or scientific value, (2) scientific validity, (3) risk minimisation and a favourable risk/benefit ratio, (4) respect for subjects, (5) informed consent, (6) independent review, (7) fair subject selection, (8) protection of confidentiality and privacy, and (9) protection of vulnerable subjects (US HHS, 2018; Emanuel et al., 2000, 2004; ICH, 1997; Resnik, 2008a). The first four criteria have significant complexity and ultimately rely on value judgements. The latter five were less difficult to meet for this research question, with clear, published guidance and therefore we do not focus on these here.

To have scientific or social value, research should lead to improvements in health, well-being or increase knowledge (Emanuel et al., 2000; World Medical Association, 2013). This research question is important because the majority of the pathways and contributors to stunting remain unexplained even though the negative effects of child 
stunting are well understood (Prendergast and Humphrey, 2014). If all proven interventions to reduce stunting were scaled up to $90 \%$ coverage, the prevalence of stunting would decrease by only 20\% (Bhutta et al., 2013). If AF is shown to contribute to linear growth faltering, interventions to reduce exposure and ultimately stunting could be developed to benefit vulnerable populations, with life-long individual and societal benefits. Additionally, this research could identify if AF acts synergistically with other stunting risk factors, such as macronutrient deficiency, further enhancing the benefits of the proposed intervention (Smith et al., 2015). We therefore concluded that this research has high scientific and social value.

A counterargument is that instead of performing research about the effect of AF on growth, resources would be better used to find ways to mitigate AF in low-resource settings and/or improve the ability of LMIC governments to enforce existing AF regulations given the known detrimental effects of AF exposure over time. We agree with the need to generate knowledge about effective adaptations of feasible interventions for LMICs and we designed a separate component of our overall research project to develop a sorting technology that could be used in local community settings. Given the high cost of control and surveillance needed to be effective in high-income countries, and the different regulatory and market structures in countries where AF persists, research and reform are needed at national and regional levels. Multi-lateral partners such as the Partnership for Aflatoxin Control in Africa (PACA) and multiple bodies working in international agricultural research work in this area. Reducing AF contamination in LMICs will require interventions at multiple levels and scales, so we believe that this research is complementary, not antagonistic or inconsistent, to the work of PACA and others.

Ethical guidelines recommend that researchers both minimise risk, provide a favourable risk-benefit ratio, and that research should only be initiated (or continued) if the anticipated benefits 'justify' or 'outweigh' the risks (US HHS, 2018; Council for International Organizations of Medical Sciences (CIOMS), 2016; ICH, 1997; World Medical Association, 2013). Beyond these principles, there is little guidance for how to calculate or make judgements of acceptable risk, nor how to assess if the risk could be exploitative to research participants (Rid and Wendler, 2011; Weijer, 2000). Exploitation not only includes exposing a research subject to unacceptable risk, but also taking unfair advantage of a subject, causing harm, perpetuating injustice or disrespect, or not providing populations or countries that participated in the research a 'fair share' of benefits (Emanuel et al., 2000; Resnik, 2003, 2008b; Wetheimer, 1996).

The question of whether AF causes stunting is only applicable to inhabitants of LMICs because those residing in high-income countries are largely protected from AF by living in regions less prone to contamination combined with their government's actions. In all possible locations for this study, and more broadly where aflatoxin continues to contaminate the food chain, maize and groundnut are dietary staples, food insecurity tends to be high and households, communities and governments do not have knowledge and/or resources to mitigate toxins, despite evidence of their deleterious health effects.

The AF problem exposes some of the vast inequalities between well-resourced and less-resourced countries and even communities within countries. Those who are at risk of AF exposure face this risk through no fault of their own, have limited mitigation options, and can't realistically migrate to avoid exposure. This situation is structurally unjust and undesirable for the half billion people around the world who are at risk. But there is an important distinction between performing research in populations who face difficult circumstances, such as poverty and food insecurity, versus taking unfair advantage of populations who are already in undesirable circumstances (Buchanan and Miller, 2006; Wetheimer, 1996). The latter would no doubt be exploitative. But research to elucidate a problem that is ongoing and disproportionately affects poor and vulnerable communities with the ambition of improving that problem for those very populations does not meet the definition of exploitation. On the contrary, the only way to improve these undesirable situations is to generate actionable evidence to remedy them.

\section{Question 2: If the relationship between $A F$ and stunting can be studied in humans, what is the most ethical study design to employ?}

Concluding that a study assessing the effect of AF on stunting with human subjects could meet ethical principles was not our endpoint, rather the beginning of multiple conversations and consultations about how to embody these principles for all research and intervention design and implementation decisions. To select a study design we compared multiple options using pre-defined criteria, considering the unique characteristics and dynamics of AF.

We ranked designs by strength of causal inference, asking (1) how well a study design could detect a change (specifically growth in this case), (2) if the participants were randomised to reduce selection bias, (3) if there is a control condition to compare outcomes, and (4) the overall likelihood that change could be attributed to the intervention (Table 1). Given that the evidence in 20142016 when these decisions were being made was mainly observational, we deemed a study design that could yield a strong casual inference essential to justify the study and increase its potential influence on the scientific community and decision-makers. We therefore considered only quasi- 
experimental (interrupted time series and controlled prepost) and experimental designs (RCT, CRT and steppedwedge design) (Bonell et al., 2011; Cousens et al., 2011; Kirkwood et al., 1997; Sanson-Fisher et al., 2014).

AF contamination in food is heterogeneous and variable within and between years. Therefore, to be scientifically valid, the design would have to control for temporal trends. Quasi-experimental designs could not sufficiently account for these. We strongly considered a stepped-wedge design, which is a one-way crossover cluster trial where all clusters eventually receive the intervention but the timing of when they receive it is randomly ordered. This design was appealing for equity reasons, as all participants would receive the same intervention. However, it would require a longer intervention period compared to an RCT or CRT, seasonality of AF exposure could remain confounding, and analysis and inference were more complicated (Hemming and Girling, 2013; Hussey and Hughes, 2007; Kotz et al., $2012,2013)$. Because we envisioned an intervention delivered through the existing health care infrastructure, we ultimately selected a CRT to generate the strongest causal inference in the shortest amount of time and with the fewest subjects.

A CRT can provide a convincing 'yes' or 'no' to our primary research question but not answer 'why' or 'why not'. We felt it would be irresponsible to invest resources into such a study with human subjects without further elucidating the 'impact pathways' of AF on growth (Kim et al., 2011; Rawat et al., 2013). Therefore, we designed a program impact pathway model and plan to link multiple data sources to these models, including survey data about infant feeding practices, blood and urine AF biomarkers and food contamination at five time points throughout the trial.

\section{Question 3: What is the most ethical intervention to randomly allocate?}

Unlike most biomedical and nutritional research that assess the efficacy of provided therapeutics, treatments and behavioural interventions, a toxin is not something that can be given to a research participant. To understand the health impact of AF through an intervention, it needs to be reduced or removed from one's environment where it is preexisting. Therefore, our operational strategy in intervention design was to reduce risk of toxin exposure to the most vulnerable member of the household (the infant), while not increasing risk exposure to others in the household or the community, to monitor infant health for signs of undernutrition, and inform all subjects about potential risks discovered in the study (described in Question 4) (Resnik et al., 2005).

Early in our planning, the lead US-based research institution formed a collaborative partnership with a Tanzanian university and researchers who study AF exposure. Together, and with consultation of local government officials in potential research sites, we performed formative research to identify specific components of our intervention. We considered the use of (1) pre- and post-harvest interventions, including biocontrol, (2) sorbing using an enterosorbent, such as NovaSil, (3) hand sorting methods to remove contaminated maize and groundnut kernels, and (4) swapping household foods consumed by infants with low-AF version of the same foods. Similar to our process for evaluating study designs, we ranked each intervention based on available literature and our experiences as researchers, by (1) intervention safety, (2) feasibility of delivery, (3) evidence to reduce AF consumption or its effects after consumption (in the case of NovaSil) and (4) likelihood of effectiveness to reduce AF in infant-consumed foods (not only household foods) over one year's time (Table 2).

Pre-harvest interventions to reduce AF include biological control, such as Aflasafe, use of genetically resistant crop varieties, chemical control and good agricultural practices, such as timely harvest and removal of crops from fields (Wild et al., 2015; Wu and Khlangwiset, 2010a). Postharvest interventions to reduce AF include drying of crops prior to storage, improved storage practices to reduce moisture and application of insecticide, and sorting (Turner et al., 2005; Wild et al., 2015; Wu and Khlangwiset, 2010a).

Table 1. Strength of causal inference for potential study designs.

\begin{tabular}{|c|c|c|c|c|}
\hline Study design & Has change occurred? & $\begin{array}{l}\text { Are participants } \\
\text { randomised? }\end{array}$ & $\begin{array}{l}\text { Is there a control } \\
\text { condition? }\end{array}$ & $\begin{array}{l}\text { Likelihood that change is } \\
\text { due to the intervention? }\end{array}$ \\
\hline Randomised controlled trial & Strong & Yes & Yes & High \\
\hline Cluster randomised controlled trial & Strong & Yes & Yes & High \\
\hline Stepped wedge design & Strong & Yes & Yes & Moderate $^{1}$ \\
\hline Interrupted time series & Moderate & No & No & Low $^{1}$ \\
\hline Controlled pre/post & Moderate & No & Yes & Low $^{1}$ \\
\hline Cohort or cross-sectional study & Moderate & No & No & Low $^{1}$ \\
\hline
\end{tabular}


Table 2. Rating of dimensions for potential trial interventions.

\begin{tabular}{|c|c|c|c|c|}
\hline Intervention & Safety & Feasibility of delivery & $\begin{array}{l}\text { Level of evidence to } \\
\text { reduce negative effects } \\
\text { of aflatoxin/aflatoxin } \\
\text { consumption in infants }\end{array}$ & $\begin{array}{l}\text { Likelihood of effectiveness } \\
\text { to reduce aflatoxin exposure } \\
\text { in infant diets for duration of } \\
\text { intervention period (one year) }\end{array}$ \\
\hline Biocontrol & Uncertain & Not feasible & None & None - low (Pitt, 2019) \\
\hline Sorbing & Limited data for infants & $\begin{array}{l}\text { Low to moderate (Wu and } \\
\text { Khlangwiset, 2010b) }\end{array}$ & High (Mitchell et al., 2014) & Unknown for long term \\
\hline Hand-sorting at household & $\begin{array}{l}\text { High concern about } \\
\text { management of toxic } \\
\text { out-sorts }\end{array}$ & $\begin{array}{l}\text { Limited evidence at large- } \\
\text { scale }\end{array}$ & Low (Xu et al., 2017) & Low \\
\hline Food swapping at household & High & $\begin{array}{l}\text { Moderate (complex } \\
\text { exchange of tons of food) }\end{array}$ & Insufficient data & Low - not targeted to infants \\
\hline $\begin{array}{l}\text { IYCF education with } \\
\text { provision and promotion of } \\
\text { infant foods }\end{array}$ & High & $\begin{array}{l}\text { Moderate (including a } \\
\text { complex but more targeted } \\
\text { food swap aimed at } \\
\text { complementary food only) }\end{array}$ & $\begin{array}{l}\text { Moderate (new intervention, } \\
\text { but designed with } \\
\text { effective pieces from other } \\
\text { nutrition and food security } \\
\text { interventions) }\end{array}$ & Moderate \\
\hline
\end{tabular}

There is evidence that many of these pre- and post-harvest interventions can be effective, but we believed them to lack the precision needed to control AF consumption for a long-term research intervention targeting infant dietary intake. Additionally, Aflasafe had not been authorised for use in Tanzania at that time. Pilot studies of community hand-sorting of groundnuts to reduce AF in communities have shown promising results, however, sustainability of the intervention in food-insecure regions and safety of the most toxic 'out-sorts' was a critical concern, as these could pose high risk to humans and animals (Filbert and Brown, 2012; Xu et al., 2017). If these out-sorts were consumed or sold, a sorting intervention may do greater harm than not sorting at all because the out-sorts concentrate the most toxic kernels. Additionally, out-sorts may be fed to and cause toxicosis to household owned livestock, to the detriment of the family's food and/or socio-economic status. We did not believe the research project or local partners could have the capacity to adequately monitor and dispose of the out-sorts from households across a district.

Sorbing has been shown to be effective in adults in shortterm studies (up to three months), but we ruled it out due to limited evidence about its safety, including micronutrient bioavailability, in infants for longer durations (Mitchell et al., 2014; Phillips et al., 2008; Wu and Khlangwiset, 2010a). As mentioned above, a separate component of our overall research project was to develop a scalable and long-term sorting technology that could be used in local mills to remove toxins in maize and groundnut kernels. However, at the start of the trial this technology was not ready for implementation. Swapping of household foods was the safest option, but for a 12-month intervention period would have been costly and perhaps not sufficiently targeted to infant diets.

We therefore developed an intervention drawing upon effective public health nutrition and food security interventions. We designed an infant and young child feeding (IYCF) educational intervention with provision (intervention arm) or promotion (control arm) of commonly consumed blended porridge flours. This IYCF intervention was created to meet multiple objectives: (1) promote a nutritionally diverse and adequate diet for all infants in the study, including exclusive breastfeeding through six months of age, (2) minimise dietary differences between intervention arms, and (3) generate strong causal evidence.

A nutritionally diverse diet, exclusive breastfeeding to six months and continued breast-feeding beyond six months are protective behaviours against moderate and high AF exposure in infants and young children (Gong et al., 2003, 2004; Wu et al., 2014b). We used these elements to design an intervention that would reduce the risk of malnutrition and AF exposure in all participants. To generate strong causal inference about AF exposure, we needed to create a contrast of AF consumption between the control and intervention groups, without creating differential macro- or micronutrient intake, nor differences in feeding and care practices that could affect stunting between arms. To reduce the risk of introducing these biases, we designed the intervention to include: (1) education to improve infant feeding and care practices using Tanzania Food and Nutrition Center's materials, and (2) behaviour change communication on the use of blended infant porridge (Table 3). Therefore, our control group condition is 'enhanced IYCF education', 
as the content and frequency of education is beyond the 'standard of care' for IYCF in the location of the study.

We carefully considered if the standard intervention should include groundnut as an infant food, as this is a potential source of AF. Some regional projects and government agencies in Tanzania have promoted groundnut as a nutritious infant food while others have recommended against its consumption because of potential for AF exposure. In more than one year of formative research in the research region we found that infants consume a monotonous, cereal-based diet with limited animalsource foods. Groundnuts are commonly consumed and culturally acceptable, with two-thirds of mothers in the region reported feeding groundnuts to their infants (E. Phillips et al., unpublished data). In this region groundnuts are one of the few sources of available and accessible protein, monounsaturated fats, fibre, several B vitamins, vitamin E and antioxidants (Mupunga et al., 2017). If we recommended against feeding groundnut, we could harm infants by creating nutritional deficiencies. Without an equally nutritionally-dense alternative to groundnuts, and after consultation with local District Nutrition Officer, we decided to include groundnuts in our intervention food and our feeding recommendations.

\section{Question 4: What is the best approach to sharing biological and food sample results with research subjects?}

A fourth major consideration of conducting this trial was if, how and when to share results of biological and food sample analyses with research participants. While there is a strong ethical imperative to share these results, there is no consistent guidance about when and how to do so. The National Bioethics Advisory Committee (NBAC) in the United States advises sharing results 'only when (a) the findings are scientifically valid and confirmed, (b) the findings have significant implications for the subject's health concerns, and (c) a course of action to ameliorate or treat those concerns is readily available.' When applying these standards to AF testing in foods and biomarkers, each of these proves challenging given current knowledge and available technologies for LMICs. Additionally, according to Tanzanian guidelines all data must be presented to government authorities first and approval sought before sharing with research participants.

Human AF biomarkers are not clinical measures with determined cut-offs to distinguish between safe or potentially unsafe levels. Without established clinical cut-offs, there is no clear scientific or health justification to flag or report results except at the levels observed in cases of acute aflatoxicosis (IARC, 2002; Williams et al., 2004). Outside of the range of acute illness, the research will undoubtedly find results that are in the upper ranges compared to other research subjects or other reported data. However, there are no established recommended actions or interventions in this putative 'grey zone'.

Secondly, there is no available technology that will allow rapid analysis of blood or urine samples in the field, so samples must be taken to a laboratory. Analysis of AF-alb requires equipment that is not available in Tanzania, so samples were to be exported for analysis, creating a time lag between sample collection and analysis. We considered working with a local laboratory to build local capacity for AF-alb, but the assays require a high level of investment and it was deemed not possible to accomplish in our budget and time frame. We did, however, built local capacity for analysis of urine biomarkers using ELISA. Even so, because

Table 3. Description of trial intervention by arm (Modified from Phillips et al., 2020).

\author{
Enhanced IYCF Education Plus Porridge Flour Provision Group \\ (Intervention) \\ Infant feeding education: \\ - Breastfeeding (exclusive through 6 months and continued through \\ 2 years) \\ - Dietary diversity \\ - Feeding frequency \\ - Hand washing
}

Behaviour change communication on the use of pre-blended porridge flours

- Timing of introduction, frequency of feeding, density and composition of porridge

- Provision of 4:1 ratio of maize meal to groundnut powder made from low-aflatoxin ingredients

- Provision of low-aflatoxin groundnut flour
Enhanced IYCF Education Group Plus Porridge Flour Promotion Group (Control)

Infant feeding education:

- Breastfeeding (exclusive through 6 months and continued through 2 years)

- Dietary diversity

- Feeding frequency

- Hand washing

Behaviour change communication on the use of pre-blended porridge flours

- Timing of introduction, frequency of feeding, density and composition of porridge

- Promotion of 4:1 ratio of maize meal to groundnut powder made from household ingredients

- Promotion of groundnut flour 
the dynamics of AF exposure are highly variable, by the time sample outliers would be identified, the properties of food consumed by the individual would undoubtedly differ from those consumed when the sample was taken. The results of the analysed samples would not be relevant by the time it could be reported.

Given that AF biomarker and food testing fail to meet the criteria for sharing results described by the NBAC and needing to first consult with the Tanzanian authorities, we designed protocols for sharing results in the case of outlying values for food and biomarker specimens. These are described in detail in our trial protocol (ClinicalTrials. gov identifier: NCT03940547). Briefly, for blood and urinary biomarker reporting, we decided to report the percent of samples above $1000 \mathrm{pg} / \mathrm{mg}$ to local authorities. We subjectively chose this cut-off because these are these are levels usually found in the highest quintile in other high-risk countries and we believed that this threshold represented our best estimate to identify this as a reporting concern. For urinary aflatoxin $M_{1}$ data, there is no evidence to determine a cut point, so we were unable to take any action based on those analyses.

The legal limit for total AF allowed in foods at the point of sale in Tanzania is $10 \mu \mathrm{g} / \mathrm{kg}$ (Anonymous, 2004). There are no set standards for AF in IYC foods in Tanzania or East Africa. We chose a limit of $5 \mu \mathrm{g} / \mathrm{kg}$ for total AF as a more stringent cut off for IYC foods in our research project. Given the documented AF problem in the region of the research site and our formative research in this location, and the significant amount of food grown and sold outside of regulated markets, we decided to respond to exceptionally high levels of AF in for food samples. We decided to report the percent $\mathrm{AF}>1000 \mu \mathrm{g} / \mathrm{kg}$ in maize or $>5,000 \mu \mathrm{g} / \mathrm{kg}$ in groundnut to the local authorities. We subjectively chose these cut-offs because these are these are levels usually found in the highest quintile in other high-risk countries and we estimate that maize is consumed five times as much as groundnut. If a second food sample from the same household were above these cut-offs we would make a home visit to the family to assess the health of the infant and understand more the food security and food quality issues in the household, particularly as they pertain to the infant and possibly refer the infants to health and agricultural authorities.

\section{Discussion and conclusions}

As researchers attempt to understand and find solutions to complex, multi-faceted problems that affect people in lowresource contexts, they must grapple with ethical questions that are unique to the type of research and the conditions and inequities of those settings. We have made our planning and decision-making processes transparent to assist other researchers when the nuances of their ethical questions are not addressed by guidelines or statements.

We can very broadly share the lessons learned through our planning experiences. Research conducted with teams of multi-disciplinary and multi-national professionals brings a wide variety of perspectives and viewpoints. The process of considering ethical aspects of this study was enhanced by having a diverse team of experts from multiple research backgrounds, and intentionally establishing a structure of multi-national leadership and oversight positions. With such a diverse group, throughout our planning process there were some areas that required multiple conversations to come to consensus. For example, we faced disagreement around if and when to report food, blood and urine results to participants given the lack of precision about their implications and limited options to sustainably remove toxins. To structure and guide our decision-making in these cases, we explored the literature about recommendations and precedents for reporting results in similar instances. Then we jointly and iteratively reviewed and discussed our protocols until there was accord.

Our funder, The Bill \& Melinda Gates Foundation, initiated this research and agreed to fund us to perform an ethical review prior to developing a full research proposal, and further conducted an external review of the final research proposal prior to its acceptance. We benefited by having a multi-year planning process to explore these decisions and flexibility in all trial and intervention design decisions. These factors allowed us to help ensure open discussions, sharing of beliefs and opinions, and building of trust among stakeholders. This approach provided transparency and objectivity in developing, running and reporting of the study.

Given the importance of stunting as a problem of global child health and the vulnerability of many families and children to chronic consumption of AF-contaminated food systems, we were strongly motivated to conduct this research. We recognise that there are no definitive answers to these questions and others might come to different conclusions. Yet we believe this research is valuable, not only to better understand the problem, but to motivate action to improve it.

\section{Acknowledgements}

This work was supported by the Bill \& Melinda Gates Foundation.

\section{Conflict of interest}

The authors declare no conflict of interest. 


\section{References}

Anonymous, 2004. Tanzanian Standard TZS 1734. Tanzanian Bureau of Standards, Dar Es Salaam, Tanzania.

Bhutta, Z.A., Das, J.K., Rizvi, A., Gaffey, M.F., Walker, N., Horton, S., Webb, P., Lartey, A., Black, R.E., The Lancet Nutrition Interventions Review Group, and the Maternal and Child Nutrition Study Group, 2013. Evidence-based interventions for improvement of maternal and child nutrition: what can be done and at what cost? The Lancet 382: 452-477. https://doi.org/10.1016/S0140-6736(13)60996-4

Bonell, C.P., Hargreaves, J., Cousens, S., Ross, D., Hayes, R., Petticrew, M. and Kirkwood, B.R., 2011. Alternatives to randomisation in the evaluation of public health interventions: design challenges and solutions. Journal of Epidemiology and Community Health 65: 582-587. https://doi.org/10.1136/jech.2008.082602

Buchanan, D.R. and Miller, F.G., 2006. Justice and fairness in the Kennedy Krieger Institute lead paint study: the ethics of public health research on less expensive, less effective interventions. American Journal of Public Health 96: 781-787. https://doi. org/10.2105/AJPH.2005.063719

Council for International Organizations of Medical Sciences (CIOMS), 2016. International Ethical Guidelines for Health-related Research Involving Humans. CIOMS, Geneva, Switzerland.

Cousens, S., Hargreaves, J., Bonell, C., Armstrong, B., Thomas, J., Kirkwood, B.R. and Hayes, R., 2011. Alternatives to randomisation in the evaluation of public-health interventions: statistical analysis and causal inference. Journal of Epidemiology and Community Health 65: 576-581. https://doi.org/10.1136/jech.2008.082610

Emanuel, E.J., Wendler, D. and Grady, C., 2000. What makes clinical research ethical? JAMA 283: 2701-2711.

Emanuel, E.J., Wendler, D., Killen, J. and Grady, C., 2004. What makes clinical research in developing countries ethical? The benchmarks of ethical research. Journal of Infectious Diseases 189: 930-937. https://doi.org/10.1086/381709

Filbert, M.E. and Brown, D.L., 2012. Aflatoxin contamination in Haitian and Kenyan peanut butter and two solutions for reducing such contamination. Journal of Hunger and Environmental Nutrition 7: 321-332. https://doi.org/10.1080/19320248.2012.707109

Gong, Y., Hounsa, A., Egal, S., Turner, P.C., Sutcliffe, A.E., Hall, A.J., Cardwell, K. and Wild, C.P., 2004. Postweaning exposure to aflatoxin results in impaired child growth: a longitudinal study in Benin, West Africa. Environmental Health Perspectives 112: 1334-1338.

Gong, Y.Y., Cardwell, K., Hounsa, A., Egal, S., Turner, P.C., Hall, A.J. and Wild, C.P., 2002. Dietary aflatoxin exposure and impaired growth in young children from Benin and Togo: cross sectional study. BMJ 325: 20-21.

Gong, Y.Y., Egal, S., Hounsa, A., Turner, P.C., Hall, A.J., Cardwell, K.F. and Wild, C.P., 2003. Determinants of aflatoxin exposure in young children from Benin and Togo, West Africa: the critical role of weaning. International Journal of Epidemiology 32: 556-562.

Hemming, K. and Girling, A., 2013. The efficiency of stepped wedge vs. cluster randomized trials: stepped wedge studies do not always require a smaller sample size. Journal of Clinical Epidemiology 66: 1427-1428. https://doi.org/10.1016/j.jclinepi.2013.07.007
Hussey, M.A. and Hughes, J.P., 2007. Design and analysis of stepped wedge cluster randomized trials. Contemporary Clinical Trials 28 : 182-191. https://doi.org/10.1016/j.cct.2006.05.007

International Agency for Research on Cancer (IARC), 2002. Aflatoxins. In: Monograph on the evaluation of carcinogenic risks to humans. Vol. 82. Some traditional herbal medicines, some mycotoxins, naphthalene and styrene. IARC, Lyon, France, pp. 171-300.

International Council for Harmonization of Technical Requirements for Registration of Pharmaceuticals for Human Use (ICH), 1997. Good Clinical Practice: Consolidated Guidance. ICH, Geneva, Switzerland.

Katz, J., 1996. The Nuremberg Code and the Nuremberg trial: a reappraisal. JAMA 276: 1662-1666. https://doi.org/10.1001/ jama.1996.03540200048030

Kim, S.S., Habicht, J.-P., Menon, P. and Stoltzfus, R.J., 2011. How do programs work to improve child nutrition? Program impact pathways of three nongovernmental organization intervention projects in the Peruvian highlands, IFPRI, Washington, DC, USA.

Kirkwood, B.R., Cousens, S.N., Victora, C.G. and de Zoysa, I., 1997. Issues in the design and interpretation of studies to evaluate the impact of community-based interventions. Tropical Medicine and International Health 2: 1022-1029.

Kotz, D., Spigt, M., Arts, I.C., Crutzen, R. and Viechtbauer, W., 2012. Use of the stepped wedge design cannot be recommended: a critical appraisal and comparison with the classic cluster randomized controlled trial design. Journal of Clinical Epidemiology 65: 12491252. https://doi.org/10.1016/j.jclinepi.2012.06.004

Kotz, D., Spigt, M., Arts, I.C., Crutzen, R. and Viechtbauer, W., 2013. The stepped wedge design does not inherently have more power than a cluster randomized controlled trial. Journal of Clinical Epidemiology 66: 1059-1060. https://doi.org/10.1016/j. jclinepi.2013.05.004

Lewis, L., Onsongo, M., Njapau, H., Schurz-Rogers, H., Luber, G., Kieszak, S., Nyamongo, J., Backer, L., Dahiye, A.M., Misore, A., DeCock, K., Rubin, C. and Group, K.A.I., 2005. Aflatoxin contamination of commercial maize products during an outbreak of acute aflatoxicosis in eastern and central Kenya. Environmental Health Perspectives 113: 1763-1767.

Mahfuz, M., Hasan, S.M.T., Alam, M.A., Das, S., Fahim, S.M., Islam, M.M., Gazi, M.A., Hossain, M., Egner, P.A., Groopman, J.D. and Ahmed, T., 2020. Aflatoxin exposure was not associated with childhood stunting: results from a birth cohort study in a resourcepoor setting of Dhaka, Bangladesh. Public Health Nutrition 24: 3361-3370. https://doi.org/10.1017/S1368980020001421

Mitchell, N.J., Hsu, H.H., Chandyo, R.K., Shrestha, B., Bodhidatta, L., Tu, Y.K., Gong, Y.Y., Egner, P.A., Ulak, M., Groopman, J.D. and Wu, F., 2017. Aflatoxin exposure during the first 36 months of life was not associated with impaired growth in Nepalese children: An extension of the MAL-ED study. PLoS ONE 12: e0172124. https:// doi.org/10.1371/journal.pone.0172124

Mitchell, N.J., Kumi, J., Aleser, M., Elmore, S.E., Rychlik, K.A., Zychowski, K.E., Romoser, A.A., Phillips, T.D. and Ankrah, N.A., 2014. Short-term safety and efficacy of calcium montmorillonite clay (UPSN) in children. American Journal of Tropical Medicine and Hygiene 91: 777-785. https://doi.org/10.4269/ajtmh.14-0093 
Mupunga, I., Mngqawa, P. and Katerere, D.R., 2017. Peanuts, aflatoxins and undernutrition in children in Sub-Saharan Africa. Nutrients 9: 1287. https://doi.org/10.3390/nu9121287

Phillips, E., Ngure, F., Smith, L.E., Makule, E., Turner, P.C., Nelson, R., Kimanya, M., Stoltzfus, R. and Kassim, N., 2020. Protocol for the trial to establish a causal linkage between mycotoxin exposure and child stunting: a cluster randomized trial. BMC Public Health 20: 598. https://doi.org/10.1186/s12889-020-08694-6

Phillips, T.D., Afriyie-Gyawu, E., Williams, J., Huebner, H., Ankrah, N.A., Ofori-Adjei, D., Jolly, P., Johnson, N., Taylor, J., MarroquinCardona, A., Xu, L., Tang, L. and Wang, J.S., 2008. Reducing human exposure to aflatoxin through the use of clay: a review. Food Additives and Contaminants. Part A 25: 134-145. https://doi. org/10.1080/02652030701567467

Pitt, J., 2019. The pros and cons of using biocontrol by competitive exclusion as a means for reducing aflatoxin in maize in Africa. World Mycotoxin Journal 12: 103-112. https://doi.org/10.3920/ WMJ2018.2410

Pitt, J., Wild, C., Baan, R., Gelderblom, W., Miller, J.D., Riley, R. and Wu, F. (eds.), 2012. Improving public health through mycotoxin control. IARC, Lyon, France.

Prendergast, A.J. and Humphrey, J.H., 2014. The stunting syndrome in developing countries. Paediatrics and International Child Health 34: 250-265. https://doi.org/10.1179/2046905514Y.0000000158

Rawat, R., Nguyen, P.H., Ali, D., Saha, K., Alayon, S., Kim, S.S., Ruel, M. and Menon, P., 2013. Learning how programs achieve their impact: embedding theory-driven process evaluation and other program learning mechanisms in alive \& thrive. Food and Nutrition Bulletin 34: S212-S225. https://doi.org/10.1177/15648265130343S207

Resnik, D.B., 2003. Exploitation in biomedical research. Theoretical Medicine and Bioethics 24: 233-259. https://doi. org/10.1023/a:1024811830049

Resnik, D.B., 2008a. Environmental health research involving human subjects: ethical issues. Environmental Health Insights 2008: 27-34.

Resnik, D.B., 2008b. Randomized controlled trials in environmental health research: ethical issues. Journal of Environmental Health 70: $28-30$

Resnik, D.B., Zeldin, D.C. and Sharp, R.R., 2005. Research on Environmental Health Interventions: Ethical Problems and Solutions. Accountability in Research 12: 69-101. https://doi. org/10.1080/08989620590957157

Rid, A. and Wendler, D., 2011. A framework for risk-benefit evaluations in biomedical research. Kennedy Institute of Ethics Journal 21: 141-179.

Sanson-Fisher, R.W., D'Este, C.A., Carey, M.L., Noble, N. and Paul, C.L., 2014. Evaluation of systems-oriented public health interventions: alternative research designs. Annual Review of Public Health 35: 9-27. https://doi.org/10.1146/annurev-publhealth-032013-182445

Smith, L.E., Prendergast, A.J., Turner, P.C., Mbuya, M.N., Mutasa, K., Kembo, G., Stoltzfus, R.J. and Sanitation Hygiene Infant Nutrition Efficacy Trial, T., 2015. The potential role of mycotoxins as a contributor to stunting in the SHINE Trial. Clinical Infectious Diseases 61 Suppl. 7: S733-S737. https://doi.org/10.1093/cid/civ849
Tesfamariam, K., De Boevre, M., Kolsteren, P., Belachew, T., Mesfin, A., De Saeger, S. and Lachat, C., 2020. Dietary mycotoxins exposure and child growth, immune system, morbidity, and mortality: a systematic literature review. Critical Reviews in Food Science and Nutrition 60: 3321-3341. https://doi.org/10.1080/10408398.2019.1685455

The National Commission for the Protection of Human Subjects of Biomedical and Behavioral Research, 1979. Ethical principles and guidelines for the protection of human subjects of research (The Belmont Report). US Department of Health, Education, and Welfare, Washington, DC, USA. Available at: https://tinyurl.com/ychnwy8m

Turner, P.C., Collinson, A.C., Cheung, Y.B., Gong, Y., Hall, A.J., Prentice, A.M. and Wild, C.P., 2007. Aflatoxin exposure in utero causes growth faltering in Gambian infants. International Journal of Epidemiology 36: 1119-1125. https://doi.org/10.1093/ije/dym122 Turner, P.C., Sylla, A., Gong, Y.Y., Diallo, M.S., Sutcliffe, A.E., Hall, A.J. and Wild, C.P., 2005. Reduction in exposure to carcinogenic aflatoxins by postharvest intervention measures in west Africa: a community-based intervention study. The Lancet 365: 1950-1956. https://doi.org/10.1016/S0140-6736(05)66661-5

US Department of Health and Human Services (US HHS), 2018. The Common Rule. Code of Federal Regulations - Title 45 Public welfare - Part 46 Protection of human subjects. US Department of Health and Human Services, Washington, DC, USA. Available at: https://tinyurl.com/4bxcjb7s

Vardon, P.J., McLaughlin, C. and Nardinelli, C., 2003. Potential economic costs of mycotoxins in the United States, Council of Agriculture, Science, and Technology, Ames, IA, USA.

Visser, M.E., Schoonees, A., Ezekiel, C.N., Randall, N.P. and Naude, C.E., 2020. Agricultural and nutritional education interventions for reducing aflatoxin exposure to improve infant and child growth in low- and middle-income countries. Cochrane Database of Systematic Reviews 4: CD013376. https://doi.org/10.1002/14651858. CD013376.pub2

Watson, S., Moore, S.E., Darboe, M.K., Chen, G., Tu, Y.K., Huang, Y.T., Eriksen, K.G., Bernstein, R.M., Prentice, A.M., Wild, C.P., $\mathrm{Xu}$, Y., Routledge, M.N. and Gong, Y.Y., 2018. Impaired growth in rural Gambian infants exposed to aflatoxin: a prospective cohort study. BMC Public Health 18: 1247. https://doi.org/10.1186/s12889018-6164-4

Weijer, C., 2000. The ethical analysis of risk. Journal of Law, Medicine and Ethics 28: 344-361. https://doi.org/10.1111/j.1748-720x.2000. tb00686.x

Wetheimer, A., 1996. Exploitation. Princeton Universtity Press, Princeton, NJ, USA.

Wild, C.P., Miller, D.J. and Groopman, J.D. (eds.), 2015. Mycotoxin control in low- and middle-income countries. IARC Working Group Reports, 9. International Agency for Research on Cancer, Lyon, France.

Williams, J.H., Phillips, T.D., Jolly, P.E., Stiles, J.K., Jolly, C.M. and Aggarwal, D., 2004. Human aflatoxicosis in developing countries: a review of toxicology, exposure, potential health consequences, and interventions. American Journal of Clinical Nutrition 80: 1106-1122. World Medical Association, 2013. WMA Declaration of Helsinki. Ethical principles for medical research involving human subjects. WMA, Ferney-Voltaire, France. Available at: https://tinyurl.com/ ycku6apm 


\section{E. Phillips et al.}

Wu, F., Groopman, J.D. and Pestka, J.J., 2014a. Public health impacts of foodborne mycotoxins. Annual Review of Food Science and Technology 5: 351-372. https://doi.org/10.1146/annurevfood-030713-092431

Wu, F. and Khlangwiset, P., 2010a. Evaluating the technical feasibility of aflatoxin risk reduction strategies in Africa. Food Additives and Contaminants Part A 27: 658-676. https://doi. org/10.1080/19440041003639582

Wu, F. and Khlangwiset, P., 2010b. Health economic impacts and cost-effectiveness of aflatoxin-reduction strategies in Africa: case studies in biocontrol and post-harvest interventions. Food Additives \& Contaminants Part A 27: 496-509. https://doi. org/10.1080/19440040903437865
Wu, F., Mitchell, N.J., Male, D. and Kensler, T.W., 2014b. Reduced foodborne toxin exposure is a benefit of improving dietary diversity. Toxicological Sciences 141: 329-334. https://doi.org/10.1093/toxsci/ kfu137

Xu, Y.A., Doel, A., Watson, S., Routledge, M.N., Elliott, C.T., Moore, S.E. and Gong, Y.Y., 2017. Study of an educational hand sorting intervention for reducing aflatoxin B1 in groundnuts in rural Gambia. Journal of Food Protection 80: 44-49. https://doi. org/10.4315/0362-028X.JFP-16-152 